\title{
(W)WILEY
}

\section{Switching from linear to macrocyclic gadolinium-based contrast agents halts the relative T1-weighted signal increase in deep gray matter of children with brain tumors: a retrospective study}

\begin{tabular}{|r|l|}
\hline Journal: & Journal of Magnetic Resonance Imaging \\
\hline Manuscript ID & JMRI-19-0280.R1 \\
\hline Wiley - Manuscript type: & Original Research \\
\hline Classification: & $\begin{array}{l}\text { Contrast enhancement techniques < Imaging Principles and Education }< \\
\text { Basic Science, Safety and biohazards < Imaging technology and safety }< \\
\text { Basic Science, Imaging techniques and processing < Imaging technology } \\
\text { and safety < Basic Science, Contrast enhancement (head and neck) } \\
\text { Head and neck imaging < Clinical Science }\end{array}$ \\
\hline Manuscript Keywords: & contrast agent, gd deposition, brain, pediatrics, mri \\
\hline
\end{tabular}

\section{SCHOLARONE Manuscripts}


Switching from linear to macrocyclic gadolinium-based contrast agents halts the relative T1weighted signal increase in deep gray matter of children with brain tumors: a retrospective study

Selene K Rowe ${ }^{1} \mathrm{MSc}$, Daniel Rodriguez ${ }^{2} \mathrm{PhD}$, Ellie Cohen ${ }^{1} \mathrm{BSc}$, Richard Grundy ${ }^{3} \mathrm{MD}$ PhD, Paul S Morgan² PhD, Tim Jaspan ${ }^{1} \mathrm{MD}$, Robert A Dineen ${ }^{1} \mathrm{PhD}$.

${ }^{1}$ Radiology, Nottingham University Hospitals, Queen's Medical Centre, Nottingham, UK.

2 Medical Physics and Clinical Engineering, Nottingham University Hospitals, Queen's Medical Centre, Nottingham, UK.

${ }^{3}$ Children's Brain Tumour Research Centre, Nottingham University Hospitals, Queen's Medical Centre, Nottingham, UK.

Corresponding author:

Daniel Rodriguez, Medical Physics, Nottingham University Hospitals NHS Trust, Derby Road, Nottingham NG7 2UH, United Kingdom.

daniel.rodriguez@nuh.nhs.uk

Selene Rowe and Daniel Rodriguez contributed equally as first authors of this manuscript

Running Title

Gd T1w increase in children's brain 


\section{ABSTRACT}

BACKGROUND: Studies have shown signal intensity (SI) changes in brains of children exposed to repeated doses of gadolinium-based contrast agent (GBCA). HYPOTHESIS: Trajectory of changes in relative dentate nucleus (DN) and globus pallidus (GP) SI in children receiving multiple doses of GBCA will alter when switched from linear to macrocyclic agents. STUDY TYPE: retrospective longitudinal. POPULATION: 35 children, age range [0.5-17.0] years, undergoing brain tumor follow-up between 2006 and 2017. FIELD STRENGTH/SEQUENCE: Unenhanced T1WI, serial scans at both 1.5 and 3 Tesla. ASSESSMENT: Regions-of-interest were drawn on DN, GP, and SI's normalized to middle cerebellar peduncle (DN/MCP) and cerebral white-matter (GP/CWM) respectively. Change in SI ratios as a function of dose (slope gradient) calculated according to type of contrast agent received: linear only, macrocyclic only, or switchover from linear to macrocyclic. For the latter, gradients were compared before and after switchover. The effect of anticancer treatment on slope gradient was tested. STATISTICAL TESTS: One-sample t- or MannWhitney U- tests for slope gradients differing from zero. Independent samples t-tests to compare slope gradient groups. Paired sample t-tests to compare slope gradients before and after switchover. RESULTS: A significant $(p<0.05)$ increase in SI ratio was observed following multiple doses of linear but not macrocyclic agents: mean percentage increase per dose in SI was $0.063 \%$ vs $-0.034 \%$ for DN/MCP, and $0.078 \%$ vs $0.004 \%$ for GP/CWM ratios. A significant $(p<0.05)$ change of SI trajectory in DN/MCP ratio was demonstrated when switching from linear to macrocyclic agent. There was no difference in SI trajectory between patients who had anticancer therapies and those who did not, DN/MCP $p=0.740 ;$ GP/BWM $p=0.694$. DATA CONCLUSION: Switching from linear to macrocyclic gadolinium-based 
contrast agents seems to halt relative T1 signal increase in deep gray-matter in children. Anticancer treatments appeared to have no impact on trajectory of T1 SI.

\section{Keywords}

Contrast agent, gd deposition, brain, pediatrics, mri

\section{INTRODUCTION}

The first intravenous gadolinium-based contrast agent (GBCA) for MR Imaging was licensed globally for clinical use in 1988 (1). Apparent deposition of gadolinium in the brain following repeated doses of GBCA was recognized in 2014, by Kanda et al (2). The authors reported a correlation between increased signal intensity within the dentate nucleus (DN) and globus pallidus (GP) and the number of doses of GBCA received.

Several studies have shown signal intensity changes in the brains of children exposed to repeated doses of $\operatorname{GBCAs}(3,4,13,5-12)$. This is important because pediatric patients (assuming a normal life expectancy) have a longer period over which neurotoxicity can act or become manifest and be more likely to receive further doses of gadolinium throughout their lifetime.

Relative T1 hyperintensity in the DN has also been attributed to the treatment effects of chemotherapy or radiation-therapy (13) A recent study reported changes in signal intensity in the DN and GP in children with brain tumors undergoing brain irradiation that are independent of the administration of GBCA (14) and another suggests increased R1 values in adults undergoing brain irradiation may increase susceptibility to gadolinium deposition (15).

The primary hypothesis for this study is that the trajectory of progressive changes in relative DN and GP signal intensity in children receiving multiple doses of GBCA for brain tumor follow-up will change when the child is switched from a linear to a macrocyclic GBCA. 
Testing of this hypothesis by retrospective analysis has been enabled by a change in institutional GBCA administration policy. Until 2009 pediatric patients at our institution were administered the linear GBCA gadopentetate dimeglumine (Magnevist, Bayer Healthcare, Berlin, Germany) because this was the only contrast agent licensed for use in young children in the UK. In 2009 the macrocyclic agent gadobutrol, (Gadovist, Bayer Healthcare, Berlin, Germany) was licensed in the UK for children over seven years old. In 2012 it was licensed for children of 2 years old and above and in 2015 it was licensed for children less than two years of age (16). Our institution followed these timelines so that where contrast agent was administered the patient would have received either linear or macrocyclic GBCA, depending on the date of the scan and the age of the child. Over the period of the retrospective analysis the MR imagers used for scanning pediatric patients and the basic MRI sequence protocol for pediatric brain tumor evaluation remained unchanged.

Given the uncertainty about the relationship between treatment effects on deep gray matter signal intensity in children receiving multiple doses of GBCA, we test a secondary hypothesis that children receiving chemotherapy and/or radiation therapy will show a steeper trajectory of relative T1-weighted signal increases in the DN and GP than children receiving no active treatment or surgery alone. An exploratory analysis of the effect of GBCA type (linear or macrocyclic) on relative T1-weighted signal trajectories for these two patient groups is also conducted.

\section{MATERIALS AND METHODS}

Consent was obtained from the parent/guardian of all participants included in this study for their clinical data to be collated and analyzed as part of an on-going research study approved by the UK National Health Service Research Ethics Committee (04/MRE04/41). 
Participants were pediatric brain tumor patients treated at the Nottingham University NHS Trust and imaged between 2006 and 2017. Participants were included in the analysis if they had undergone MRI with 6 doses or more of either linear GBCA, macrocyclic GBCA or both, with an MRI protocol that included an unenhanced axial T1WI sequence and were under 18 years of age at the time of their first scan. Potential participants were excluded if they had a condition known to be associated with abnormal DN or GP signal on MRI; if they had brain lesions or surgical resection involving the DN or GP; or if they had previously undergone MRI outside our institution with a GBCA that could not be identified. Individual MR scans were excluded from the analysis if they originated from other institutions, did not include an unenhanced axial T1WI and if degraded by either patient motion or flow artifacts obscuring the anatomical structure of interest. A history of chemotherapy or radiation therapy administration was obtained from the clinical notes. Screening of the patients prior to their MRI scan should have ensured that none of the patients had renal impairment; however nuclear medicine and blood test results were also checked. Medical records were checked to identify any other reasons for exclusion as were their series of MRI scans.

Thirty five patients were analyzed in total. For the purposes of analysis, 3 participant sub-groups were defined based on GBCA administration: (1) The 'linear-only' group includes all those who received 6 or more doses of gadopentetate dimeglumine, but the analyzes referring to this group include only the signal intensity ratios prior to their first dose of gadobutrol (if given); (2) the 'macrocyclic-only group' includes participants who only received gadobutrol (i.e. no exposure to gadopentetate dimeglumine) and received 6 or more doses of gadobutrol; (3) the 'switchover group' included those who received 6 or more doses of gadopentetate dimeglumine followed by 3 or more doses of gadobutrol (and could therefore 
include patients already in the linear-only group). Evolution of the different groups is described in Figure 1.

\section{MR Imaging Protocol}

Scanning was performed on three scanners: an Intera 1.5 Tesla (Philips Healthcare, Best, The Netherlands); an Achieva 3 Tesla (Philips Healthcare, Best, The Netherlands) and a Signa Excite 1.5 Tesla (General Electric Healthcare, Chicago, IL, USA). Our institution uses a set protocol which was employed to scan all pediatric brain tumor patients throughout the study period to maintain consistent parameters. Parameters for the axial T1WI spin echo sequence used for analysis are shown in Table 1. The GBCA dose was calculated strictly as per the manufacturers' recommendation: $0.2 \mathrm{ml}(0.002 \mathrm{mmol} / \mathrm{ml})$ per $\mathrm{kg}$ of weight for gadopentetate dimeglumine and $0.1 \mathrm{ml}(1.0 \mathrm{mmol} / \mathrm{ml})$ per $\mathrm{kg}$ for gadobutrol. The GBCA type and dose at each time point were recorded in and collected from our institution's radiology information system.

\section{Image Analysis}

Image viewing and analysis was performed using the GE Centricity Universal Viewer V6.0 PACS system (GE Healthcare, Chicago, IL, USA). The axial T1WI sets were scored for quality using a scoring range of $0-3$. Images that were scored 0 were rejected from analysis. A second independent reviewer (neuroradiologist with 14 years' experience, RD) reviewed all included image slices to confirm that the images were appropriate for inclusion in the analysis. Regions of Interest (ROIs) were drawn manually by SR on the unenhanced axial T1WI, supplemented by reference to other $\mathrm{T} 2 \mathrm{WI}$ to aid structure identification if required. The middle cerebellar peduncle $(\mathrm{MCP})$ and the cerebral white matter (CWM) were selected as control regions for 
ratio calculations based on the relative lack of evidence of gadolinium deposition in white matter compared to deep gray matter (17-19). An elliptical ROI was placed on the DN and GP. ROIs were standardized to between 0.3 and $0.6 \mathrm{~cm}$ in size depending on the size of the anatomy. Training and supervision by a neuroradiologist with 30 years' experience, TJ, assisted in the identification of the individual structures and ensured accurate placement of ROIs. An example of the ROls drawn can be seen in Figure 2.

Ratios of mean signal intensity were calculated for the DN relative to the MCP (DN/MCP) and for the GP relative to the CWM (GP/CWM). Intensity ratios were used to reduce confounding effects from the variation in data acquisition across different scanners and field strengths. For each subject, the ratios were normalized to their respective ratio at the first scan, and the gradient of the relative signal intensity ratio slope as the number of doses increased was calculated from linear regression (Microsoft Excel 2010, Redmond, WA, USA).

For the Switchover group, ratios were calculated for before, and after contrast agent switch. Gadopentetate dimeglumine ratios at each time point were normalized by their first scan values, and gadobutrol ratios by the last unenhanced T1WI, which was acquired immediately prior to the first dose of gadobutrol.

\section{Statistical Analysis}

Statistical analysis was performed using SPSS version 21 (IBM SPSS Statistics for Windows, Version 21.0. Armonk, NY, USA). A one-sample t-tests or Mann-Whitney U tests, depending on normality, were performed to test for slope gradients differing from zero. Group comparisons of signal intensity ratio slope gradients were made using independent samples t-tests. In the switchover group, differences between slopes before and after changing the 
contrast agent were compared using paired sample t-tests. $\mathrm{P}$ values below 0.05 were considered statistically significant.

\section{RESULTS}

Of the 65 patients identified as suitable subjects, whose parents / care takers had consented to data collection, 35 individuals fulfilled the inclusion criteria for this analysis and 28 were excluded based on the exclusion criteria. A further two potential participants had individual scans without unenhanced T1WIs which put them below the required number of scans for inclusion. Out of the 35 patients selected, it was possible to analyze both the DN and GP in 18 subjects, only DN in 8 subjects and only GP in 9 subjects. This was because some had lesions/surgery in one area but not the other. Details of the 35 included participants are shown in Table 2.

A subgroup 18 patients that were scanned more 3 or more times each at 1.5 and 3 Tesla using the same contrast agent. The agreement of ratios calculated from 1.5 Tesla images only compared to those calculated from 3 Tesla images only can be seen in the Bland-Altman plot of Figure 3.

For the whole group, positive slope gradients were observed with the distribution of slope gradients varying significantly from 'no slope' for both DN/MCP (mean \pm standard deviation $(S D)=0.002 \pm 0.005, p=0.026, t=2.360)$ and $G P / C W M$, (mean $\pm S D=0.004 \pm 0.005 p$ $<0.005, t=4.271$. The mean percentage increase in signal intensity per dose across the group was $0.013 \% \pm 0.043(\mathrm{SD})$, for the $\mathrm{DN} / \mathrm{MCP}$ ratio and $0.027 \% \pm 0.041$ for the GP/CWM ratio.

The data was then split into the 'linear only' and 'macrocyclic only' groups. Analysis of the linear group showed positive slope gradients with the distribution of slope gradients varying significantly from 'no slope' for both $D N / M C P$ (mean $\pm S D=0.007 \pm 0.004, p<0.005, t$ 
$=8.618)$ and GP/CWM ( $<<0.005, t=6.07$ ) (see Figure 4a for an example of a linear group patient). The mean percentage increase per dose in signal intensity across the group was $0.063 \% \pm 0.044$ for the DN/MCP ratio, and $0.078 \% \pm 0.082$.

In contrast, the macrocyclic group showed no clear slope, confirmed by the lack of significant difference from the 'no-slope' condition in the one-sample t-test for either DN/MCP $(p=0.104, t=-1.870)$ or GP/CWM $(p=0.546, t=0.628)$ (see Figure $4 b$ for an example of a macrocyclic group patient). The mean percentage increase per dose in signal intensity across the group was $-0.034 \% \pm 0.051$ for the $\mathrm{DN} / \mathrm{MCP}$ ratio and $0.004 \% \pm 0.040$ for the GP/CWM.

For the whole group an independent samples t-test demonstrated no significant difference in the T1WI signal intensity ratio slope gradient between those who did and those who did not receive chemotherapy or radiation therapy, for neither DN/MCP or GP/CWM ratios (DN/MCP $p=0.740, t=-0.337 ; G P / B W M p=0.694, t=0.399)$. The percentage increase per dose for the whole group was $0.011 \% \pm 0.043$ for the DN/CWM and $0.024 \% \pm 0.043$. When the analysis was repeated for the linear group separately, the independent Mann-Whitney $U$ test again showed no significant difference between those who did and those who did not receive chemotherapy and/or radiation therapy (linear group: DN/MCP $p=0.246, \mathrm{GP} / \mathrm{CWM} p$ =0.733; macrocyclic group: DN/MCP $p=0.385, G P / C W M p=0.703)$. For the patients who received no therapy and were in the linear group the percentage increase per dose was $0.092 \% \pm 0.035$ for the DN/CWM and $0.137 \% \pm 0.113$ for the GP/CWM ratio. Those who had received therapy and were in the linear group showed a percentage increase per dose of $0.044 \% \pm 0.039$ for the DN/CWM ratio and $0.045 \% \pm 0.030$ for the GP/CWM ratios.

For the Switchover group, the paired samples t-test showed a significant difference in the distribution of slope gradients before and after the switch from linear to macrocyclic for 
DN/MCP ( $p=0.014)$, with the plot demonstrating an apparent reversal from increasing to decreasing trajectory of ratios at the point of switch (Figure 5a). No significant difference was seen in the distribution of slope gradients before and after the switch for GP/CWM ( $p=0.680)$, however inspection of the plot reveals a plateauing of the trajectory of ratios at the point of the switch (Figure 5b) but a noticeably larger standard deviation.

\section{DISCUSSION}

We hypothesized that the trajectory of progressive changes in relative DN and GP signal intensity in children receiving multiple doses of GBCA will change when switching from a linear to a macrocyclic GBCA. This hypothesis is supported with regard to the DN/MCP ratios, where a significant difference in slope gradients was identified in children before and after the switchover. Two other studies in adults have evaluated serial linear and subsequent macrocyclic doses in the same patients with similar findings $(20,21)$. Although no statistically significant change in trajectory was found for the GP/CWM ratios before and after the switchover, the plot of mean values shows a plateauing of the trajectory after the switch to macrocyclic GBCA.

Our data for the separate linear and macrocyclic groups also demonstrates different trajectories of signal change; the linear group showed positive slope gradients in keeping with presumed gadolinium deposition at both sites (DN and GP), whereas the macrocyclic group did not. The findings are in line with the majority of recent literature identifying increase in T1-weighted hyperintensity in the GP and DN after serial doses of linear GBCAs but not macrocyclic GBCAs $(2,4-7,11,13,22,23)$.

A notable finding was the negative slope gradient for DN/MCP ratios in the switchover group after the change to macrocyclic GBCA. This finding agrees with the two studies in adults 
that made use of a change in the type of contrast agent $(20,21)$. It is difficult to specify the reason for this decline. It has been demonstrated that macrocyclic GBCAs also deposit in the brain but to a lesser extent than linear agents (24) and few studies have reliably demonstrated signal intensity ratio increases in the macrocyclic group (25). Studies in rats demonstrated that gadolinium found in the rat brain after linear and macrocyclic contrast administration was in three distinct forms, intact GBCA, soluble macromolecules and in insoluble form, the intact GBCA being identified as macrocyclic contrast $(26,27)$. This suggests that the reason for the decline in signal intensity ratio therefore could relate to a steady washout of the linear gadolinium, which has been demonstrated in rats (28). Changes to the binding of the gadolinium; dechelation and precipitation of the gadolinium is another consideration (29). Alternatively, this decline in ratio values could be due to a disproportionate increase in signal intensity of the control ROI used in the ratio calculation, the MCP. This latter point highlights a problem with much of the existing literature that uses signal intensity ratios; i.e. the assumption that the control ROI is not subject to gadolinium deposition. For example, several previous studies use the pons as a control $\operatorname{ROI}$ for the $\operatorname{DN}(2,7,8,10,11,17,23)$, but it is recognized that the pons, which contains many gray matter nuclei, is a site of gadolinium deposition itself (13). Our data do not allow isolation of the separate effects of potential gadolinium clearance from the DN and gadolinium deposition in the MCP to be disentangled, for which quantitative T1-mapping would be required.

The values for DN/MCP and GP/CWM increased with GBCA dose number for our pediatric cohort as a whole, which broadly confirms the findings of other groups studying pediatric populations $(3,4,6,8-14)$. However, we found no significant difference in the trajectory of signal intensity ratio increase between children treated with chemotherapy and radiation therapy and those who received no treatment or surgery alone. These findings are 
in line with another study in adults and children which found that radiation therapy had no impact on signal intensity change (5), but conflict with a recent study showing that signal intensity changes in the DN and GP occur in patients with brain tumors undergoing brain irradiation independent of GBCA administration (14).

Our analysis is limited by a relatively small sample size, particularly when the sample is split into groups for analysis. It would not be ethical to conduct a prospective trial testing the relative gadolinium deposition rates in children following multiple GBCA doses, and hence we were limited to conducting a retrospective analysis. One strength of our study is that during the study period (2006 to 2017) the MRI scanners used at our institution for pediatric brain tumor evaluation, and the basic MRI sequence protocol, did not change which provides continuity in the dataset. Although patients were scanned on both 1.5 and 3 Tesla scanners, the use of signal intensity ratios removes confounding effects.

To date, brain deposition of gadolinium has not been shown to be harmful in the majority of patients and the long-term consequences are unknown. Our data do not allow us to explore the consequences of presumed gadolinium deposition for the children involved. Apart from the fact that we do not have quantitative cognitive or neurological function scores for these children, this group is likely to carry a significant neurological and cognitive burden due to their complex neurosurgical and treatment histories. In addition, maturation of brain structure and function occurs throughout childhood and hence gadolinium deposition may affect the developing brain differently to a mature adult brain. However, it is important that the consequences of gadolinium deposition are studied prospectively as detrimental effects could be greater in the developing brain that is undergoing rapid structural and functional maturation compared to the mature adult brain. 
In conclusion our analyzes confirm previous reports that T1 signal increase occurs in the deep gray matter following repeated doses of linear GBCA but not macrocyclic agents. Furthermore, we demonstrate that switching from linear to macrocyclic GBCAs halts the relative $\mathrm{T} 1$ signal increase in deep gray matter of children with brain tumors. We found no evidence of an independent effect of chemotherapy or radiation therapy on the trajectory of T1 signal increase. 


\section{References}

1. Pierre VC, Allen MJ, Caravan P: Contrast agents for MRI: 30+ years and where are we going? J Biol Inorg Chem 2015; 19:127-131.

2. Kanda T, Ishii K, Kawaguchi H, Kitajima K, Takenaka D: High signal intensity in the dentate nucleus and globus pallidus on unenhanced T1-weighted MR images: relationship with increasing cumulative dose of a gadolinium-based contrast material. Radiology 2014; 270:834-41.

3. Morcos SK: Extracellular gadolinium contrast agents: Differences in stability. Eur J Radiol $2008 ; 66: 175-179$.

4. Miller JH, Hu HH, Pokorney A, Cornejo P, Towbin R: MRI Brain Signal Intensity Changes of a Child During the Course of 35 Gadolinium Contrast Examinations. Pediatrics 2015; 136:e1637-40.

5. Adin ME, Kleinberg L, Vaidya D, Zan E, Mirbagheri S, Yousem DM: Hyperintense Dentate Nuclei on T1-Weighted MRI: Relation to Repeat Gadolinium Administration. AJNR Am J Neuroradiol 2016; 36:1859-1865.

6. Roberts DR, Chatterjee AR, Yazdani M, et al.: Pediatric Patients Demonstrate Progressive T1-Weighted Hyperintensity in the Dentate Nucleus following Multiple Doses of GadoliniumBased Contrast Agent. AJNR Am J Neuroradiol 2016; 37:2340-47.

7. Hu HH, Pokorney A, Towbin RB, Miller JH: Increased signal intensities in the dentate nucleus and globus pallidus on unenhanced T1-weighted images : evidence in children undergoing multiple gadolinium MRI exams. Pediatr Radiol 2016; 46:1590-1598.

8. Tibussek D, Rademacher C, Caspers J, et al.: Gadolinium Brain Deposition after Macrocyclic Gadolinium Administration: A Pediatric Case- Control Study. Radiology 2017; 285:223-230. 
9. Flood TF, Stence N V, Maloney JA, Mirsky DM: Pediatric Brain : Repeated Exposure to Linear Gadolinium-based Contrast Material Is Associated with Increased Signal Intensity at Unenhanced T1- weighted MR Imaging. Radiology 2017; 282:222-228.

10. Rossi Espagnet MC, Bernardi B, Pasquini L, Figà-Talamanca L, Tomà P, Napolitano A: Signal intensity at unenhanced T1-weighted magnetic resonance in the globus pallidus and dentate nucleus after serial administrations of a macrocyclic gadolinium-based contrast agent in children. Pediatr Radiol 2017; 47:1345-1352.

11. Radbruch A, Haase R, Kickingereder P, et al.: Pediatric Brain : No Increased Signal Intensity in the Dentate Nucleus on Unenhanced T1-weighted MR Images after Consecutive Exposure to a Macrocyclic Gadolinium-based Contrast Agent. Radiology 2017; 283:828-836. 12. Young J, Orosz I, Franke M, et al.: Gadolinium deposition in the paediatric brain: T1weighted hyperintensity within the dentate nucleus following repeated gadolinium-based contrast agent administration. Clin Radiol 2018; 73:290-295.

13. Kasahara S, Miki Y, Kanagaki M, et al.: Hyperintense Dentate Nucleus on Unenhanced T1-weighted MR Images Is Associated with a History of Brain Irradiation. Radiology 2011; 258:222-8.

14. Tamrazi B, Nguyen B, Liu C, et al.: Changes in Signal Intensity of the Dentate Nucleus and Globus Pallidus in Pediatric Patients: Impact of Brain Irradiation and Presence of Primary Brain Tumors Independent of Linear Gadolinium- based Contrast Agent Administration. Radiology 2018; 287:452-460.

15. Lim WH, Choi SH, Yoo R, et al.: Does radiation therapy increase gadolinium accumulation in the brain ?: Quantitative analysis of T1 shortening using R1 relaxometry in glioblastoma multiforme patients. PLoS One 2018; 13.

16. Schwartz GJ, Work DF: Measurement and Estimation of GFR in Children and Adolescents. 
Clin J Am Soc Nephrol 2009; 4:1832-1843.

17. Mcdonald RJ, Mcdonald JS, Kallmes DF, et al.: Intracranial Gadolinium Deposition after Contrast-enhanced MR Imaging. Radiology 2015; 275:772-82.

18. Murata N, Murata K, Gonzalez-cuyar LF, Maravilla KR: Gadolinium tissue deposition in brain and bone. Magn Reson Imaging 2016; 34:1359-1365.

19. Kanda T, Fukusato T, Matsuda M, et al.: Gadolinium-based Contrast Agent Accumulates in the Brain Even in Subjects without Severe Renal Dysfunction: Evaluation of Autopsy Brain Specimens with Inductively Coupled Plasma Mass Spectroscopy. Radiology 2015; 276:22832.

20. Radbruch A, Weberling LD, Kieslich PJ, et al.: Intraindividual Analysis of Signal Intensity Changes in the Dentate Nucleus After Consecutive Serial Applications of Linear and Macrocyclic Gadolinium-Based Contrast Agents. Invest Radiol 2016; 51:683-690.

21. Behzadi A, Farooq Z, Zhao Y, Shih G, Prince M: Dentate Nucleus Signal Intensity Decrease on T1-weighted MR Images after Switching from Gadopentetate Dimeglumine to Gadobutrol. Radiology 2018; 287:816-823.

22. Radbruch A, Weberling L, Kieslich P, et al.: Gadolinium Retention in the Dentate Nucleus and Globus Pallidus Is Dependent on the Class of Contrast Agent. Radiology 2015; 275:783791.

23. Eisele $\mathrm{P}$, Alonso A, Szabo K, et al.: Lack of increased signal intensity in the dentate nucleus after repeated administration of a macrocyclic contrast agent in multiple sclerosis. Medicine (Baltimore) 2016; 95.

24. Murata N, Gonzalez-Cuyar L, Murata K, et al.: Macrocyclic and Other Non - Group 1 Gadolinium Contrast Agents Deposit Low Levels of Gadolinium in Brain and Bone Tissue Preliminary Results From 9 Patients With Normal Renal Function. Invest Radiol 2016; 
51:447-53.

25. Stojanov DA, Aracki-trenkic A, Vojinovic S, Benedeto-stojanov D: Increasing signal intensity within the dentate nucleus and globus pallidus on unenhanced T1W magnetic resonance images in patients with relapsing-remitting multiple sclerosis : correlation with cumulative dose of a macrocyclic gadolinium-based contrast ag. Eur Radiol 2016; 26:807815.

26. Frenzel T, Apte C, Jost G, Schöckel L, Lohrke J, Pietsch H: Quantification and Assessment of the Chemical Form of Residual Gadolinium in the Brain After Repeated Administration of Gadolinium-Based Contrast Agents Comparative Study in Rats. Invest Radiol 2017; 52:18-20.

27. Robert P, Fingerhut S, Factor MSC, Vives V, Letien BSJ: One-year Retention of Gadolinium in the Brain : Comparison of Gadodiamide and Gadoterate Meglumine in a Rodent Model. Radiology 2018; 288:424-433.

28. Smith APL, Marino M, Crowder JM, et al.: Clearance of Gadolinium from the Brain with No Pathologic Effect after Repeated Administration of Gadodiamide in Healthy Rats: An Analytical and Histologic Study. Radiology 2017; 282:743-751.

29. Gianolio E, Bardini P, Arena F, et al.: Gadolinium Retention in the Rat Brain : Assessment of the Amounts of Insoluble Gadolinium-containing Species and Intact Gadolinium Complexes after Repeated Administration of Gadolinium-based Contrast Agents. Radiology $2017 ; 285: 839-849$. 
1. Pierre VC, Allen MJ, Caravan P. Contrast agents for MRI: $30+$ years and where are we going? J Biol Inorg Chem. 2015;19(2):127-131.

2. Kanda $\mathrm{T}$, Ishii $\mathrm{K}$, Kawaguchi $\mathrm{H}$, et al. High signal intensity in the dentate nucleus and globus pallidus on unenhanced T1-weighted MR images: relationship with increasing cumulative dose of a gadolinium-based contrast material. Radiology. $2014 ; 270(3): 834-841$.

3. Morcos SK. Extracellular gadolinium contrast agents: Differences in stability. Eur J Radiol. 2008;66:175-179.

4. Miller JH, Hu HH, Pokorney A, et al. MRI Brain Signal Intensity Changes of a Child During the Course of 35 Gadolinium Contrast Examinations. Pediatrics. 2015;136(6):e1637-40.

5. Adin ME, Kleinberg L, Vaidya D, et al. Hyperintense Dentate Nuclei on T1-Weighted MRI: Relation to Repeat Gadolinium Administration. AJNR Am J Neuroradiol. 2016;36(10):1859-1865.

6. Roberts DR, Chatterjee AR, Yazdani M, et al. Pediatric Patients Demonstrate Progressive T1-Weighted Hyperintensity in the Dentate Nucleus following Multiple Doses of Gadolinium-Based Contrast Agent. AJNR Am J Neuroradiol. 2016;37:23402347.

7. $\mathrm{Hu} \mathrm{HH}$, Pokorney A, Towbin RB, et al. Increased signal intensities in the dentate nucleus and globus pallidus on unenhanced T1-weighted images : evidence in children undergoing multiple gadolinium MRI exams. Pediatr Radiol. 2016;46:15901598.

8. Tibussek D, Rademacher C, Caspers J, et al. Gadolinium Brain Deposition after Macrocyclic Gadolinium Administration: A Pediatric Case- Control Study. Radiology. $2017 ; 285(1): 223-230$.

9. Flood TF, Stence N V, Maloney JA, et al. Pediatric Brain : Repeated Exposure to 
Linear Gadolinium-based Contrast Material Is Associated with Increased Signal Intensity at Unenhanced T1- weighted MR Imaging. Radiology. 2017;282(1):222-228.

10. Rossi Espagnet MC, Bernardi B, Pasquini L, et al. Signal intensity at unenhanced T1weighted magnetic resonance in the globus pallidus and dentate nucleus after serial administrations of a macrocyclic gadolinium-based contrast agent in children. Pediatr Radiol. 2017;47:1345-1352.

11. Radbruch A, Haase R, Kickingereder P, et al. Pediatric Brain : No Increased Signal Intensity in the Dentate Nucleus on Unenhanced T1-weighted MR Images after Consecutive Exposure to a Macrocyclic Gadolinium-based Contrast Agent. Radiology. $2017 ; 283(3): 828-836$.

12. Young J, Orosz I, Franke M, et al. Gadolinium deposition in the paediatric brain: T1weighted hyperintensity within the dentate nucleus following repeated gadoliniumbased contrast agent administration. Clin Radiol. 2018;73(3):290-295.

13. Kasahara S, Miki Y, Kanagaki M, et al. Hyperintense Dentate Nucleus on Unenhanced T1-weighted MR Images Is Associated with a History of Brain Irradiation. Radiology. 2011;258(1):222-228.

14. Tamrazi B, Nguyen B, Liu C, et al. Changes in Signal Intensity of the Dentate Nucleus and Globus Pallidus in Pediatric Patients: Impact of Brain Irradiation and Presence of Primary Brain Tumors Independent of Linear Gadolinium- based Contrast Agent Administration. Radiology. 2018;287(2):452-460.

15. Lim WH, Choi SH, Yoo R, et al. Does radiation therapy increase gadolinium accumulation in the brain ?: Quantitative analysis of T1 shortening using R1 relaxometry in glioblastoma multiforme patients. PLoS One. 2018;13(2).

16. Schwartz GJ, Work DF. Measurement and Estimation of GFR in Children and Adolescents. Clin J Am Soc Nephrol. 2009;4:1832-1843.

17. Mcdonald RJ, Mcdonald JS, Kallmes DF, et al. Intracranial Gadolinium Deposition after Contrast-enhanced MR Imaging. Radiology. 2015;275(3):772-782.

18. Murata N, Murata K, Gonzalez-cuyar LF, et al. Gadolinium tissue deposition in brain 
and bone. Magn Reson Imaging. 2016;34(10):1359-1365.

19. Kanda T, Fukusato T, Matsuda M, et al. Gadolinium-based Contrast Agent Accumulates in the Brain Even in Subjects without Severe Renal Dysfunction: Evaluation of Autopsy Brain Specimens with Inductively Coupled Plasma Mass Spectroscopy. Radiology. 2015;276(1):228-232.

20. Radbruch A, Weberling LD, Kieslich PJ, et al. Intraindividual Analysis of Signal Intensity Changes in the Dentate Nucleus After Consecutive Serial Applications of Linear and Macrocyclic Gadolinium-Based Contrast Agents. Invest Radiol. 2016;51(11):683-690.

21. Behzadi A, Farooq Z, Zhao Y, et al. Dentate Nucleus Signal Intensity Decrease on T1-weighted MR Images after Switching from Gadopentetate Dimeglumine to Gadobutrol. Radiology. 2018;287(3):816-823.

22. Radbruch A, Weberling L, Kieslich P, et al. Gadolinium Retention in the Dentate Nucleus and Globus Pallidus Is Dependent on the Class of Contrast Agent. Radiology. 2015;275(3):783-791.

23. Eisele $\mathrm{P}$, Alonso A, Szabo K, et al. Lack of increased signal intensity in the dentate nucleus after repeated administration of a macrocyclic contrast agent in multiple sclerosis. Medicine (Baltimore). 2016;95(39).

24. Murata N, Gonzalez-Cuyar L, Murata K, et al. Macrocyclic and Other Non - Group 1 Gadolinium Contrast Agents Deposit Low Levels of Gadolinium in Brain and Bone Tissue Preliminary Results From 9 Patients With Normal Renal Function. Invest Radiol. 2016;51(7):447-453.

25. Stojanov DA, Aracki-trenkic A, Vojinovic S, et al. Increasing signal intensity within the dentate nucleus and globus pallidus on unenhanced T1W magnetic resonance images in patients with relapsing-remitting multiple sclerosis : correlation with cumulative dose of a macrocyclic gadolinium-based contrast ag. Eur Radiol. 2016;26:807-815.

26. Frenzel T, Apte C, Jost G, et al. Quantification and Assessment of the Chemical Form 
of Residual Gadolinium in the Brain After Repeated Administration of Gadolinium-

Based Contrast Agents Comparative Study in Rats. Invest Radiol. 2017;52(7):18-20.

27. Robert P, Fingerhut S, Factor MSC, et al. One-year Retention of Gadolinium in the Brain : Comparison of Gadodiamide and Gadoterate Meglumine in a Rodent Model. Radiology. 2018;288(2):424-433.

28. Smith APL, Marino M, Crowder JM, et al. Clearance of Gadolinium from the Brain with No Pathologic Effect after Repeated Administration of Gadodiamide in Healthy Rats: An Analytical and Histologic Study. Radiology. 2017;282(3):743-751.

29. Gianolio E, Bardini P, Arena F, et al. Gadolinium Retention in the Rat Brain : Assessment of the Amounts of Insoluble Gadolinium-containing Species and Intact Gadolinium Complexes after Repeated Administration of Gadolinium-based Contrast Agents. Radiology. 2017;285(3):839-849. 


\section{Tables}

Table 1: Parameters for axial T1WI spin echo imaging

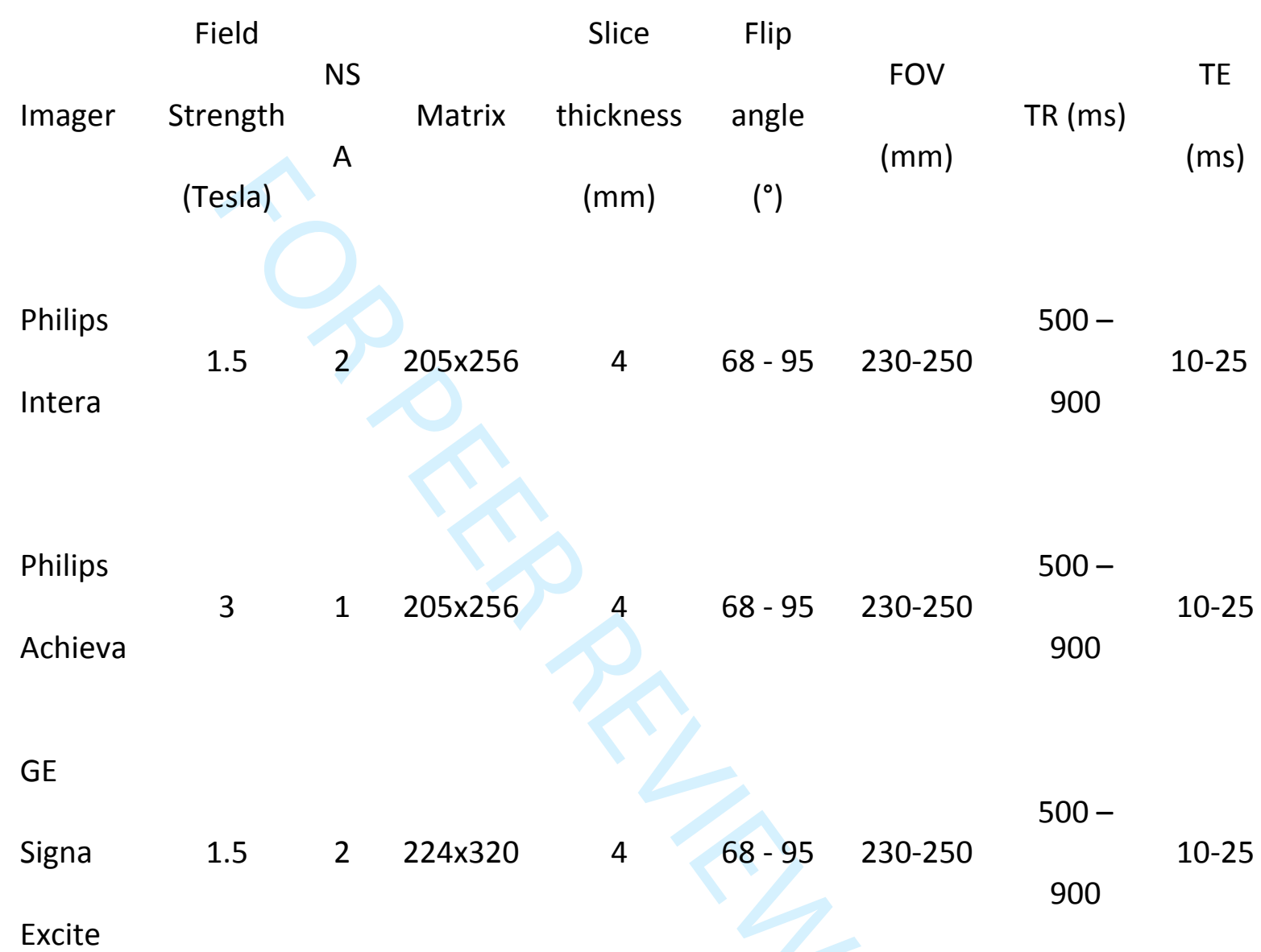

$T R=$ repetition time; $T E$ = echo time; NSA = number of signal averages; FOV = field of view 
Table 2: Characteristics of the participants included in the analysis.

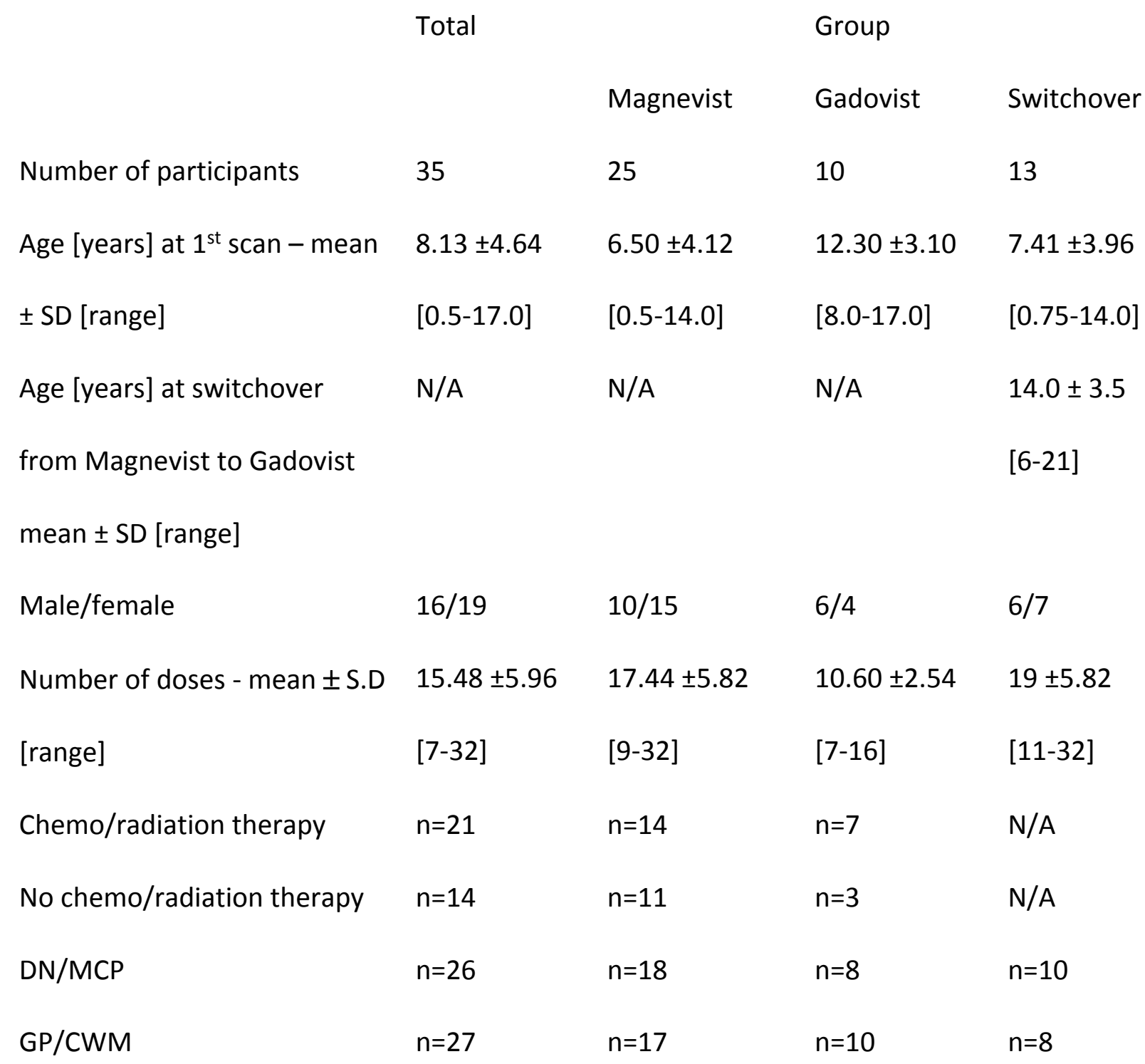

$\mathrm{SD}=$ standard deviation, $\mathrm{DN}=$ dentate nucleus, $\mathrm{GP}=$ globus pallidus, $\mathrm{MCP}=$ middle cerebellar peduncle, $\mathrm{CWM}=$ cerebral white matter . 


\section{Figure Legends}

Figure 1- Flow chart showing patient group numbers as derived from the exclusion process, the number of scans analyzed and rejected and the patients' diagnoses. $\mathrm{DN}=$ Dentate Nucleus, GP = Globus Pallidus.

Figure 2 - Examples of region of interest (ROI) placement for (a) right dentate nucleus (b) right middle cerebellar peduncle, (c) right globus pallidus, (d) cerebral white matter. All ROIs were placed on pre-contrast axial T1-weighted images.

Figure 3 - Bland-Altman plot comparing signal intensity ratio slope gradients for patients that were scanned more 3 or more times each at 1.5 and 3 Tesla using the same contrast agent. Apart from 2 outliers, all repeated measurements are within the $95 \%$ limits of agreement, and most present very small differences when measured with either a 1.5 or a 3 Tesla scanner.

Figure 4-Individual subject data for relative globus pallidus (GP) / cerebral white matter (CWM) T1 signal intensity ratios for (a) a patient from the linear group and (b) a patient from the macrocyclic group. DN/MCP and GP/CWM ratios for each dose are expressed relative to the respective values derived from the unenhanced T1-weighted image prior to the first dose of gadolinium based contrast agent (GBCA).

Figure 5 - Group mean values $(n=18)$ for each dose and fitted slopes from the switchover group for the relative (a) dentate nucleus (DN) / middle cerebellar peduncle (MCP) and (b) 
globus pallidus (GP) / cerebral white matter (CWM) T1 signal intensity ratios before (dashed line) and after (dash-dot line) the switch from linear to macrocyclic. For each patient, the intensity ratios of the linear group are normalized by the ratio of the last linear contrast agent scan, and macrocyclic ratios by the ratio of their first macrocyclic scan, so that both those scans are plotted at $(0,1)$. The gray area represents the standard deviation around the mean values. 
Flow chart showing patient group numbers as derived from the exclusion process, the number of scans analyzed and rejected and the patients' diagnoses. DN = Dentate Nucleus, GP = Globus Pallidus.

$56 \times 52 \mathrm{~mm}(300 \times 300 \mathrm{DPI})$ 


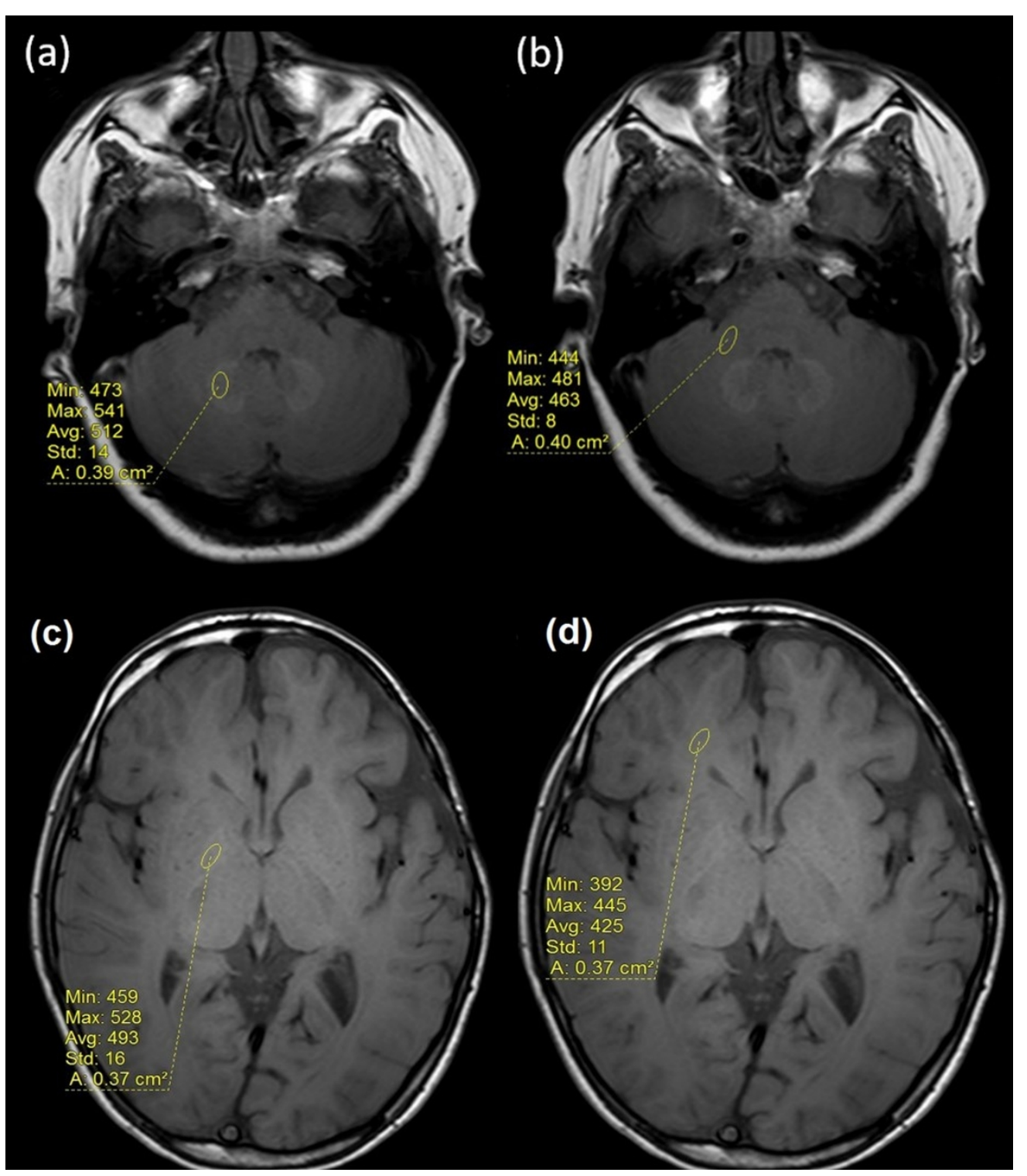

Examples of region of interest (ROI) placement for (a) right dentate nucleus (b) right middle cerebellar peduncle, (c) right globus pallidus, (d) cerebral white matter. All ROIs were placed on pre-contrast axial T1weighted images.

$101 \times 116 \mathrm{~mm}(600 \times 600 \mathrm{DPI})$ 


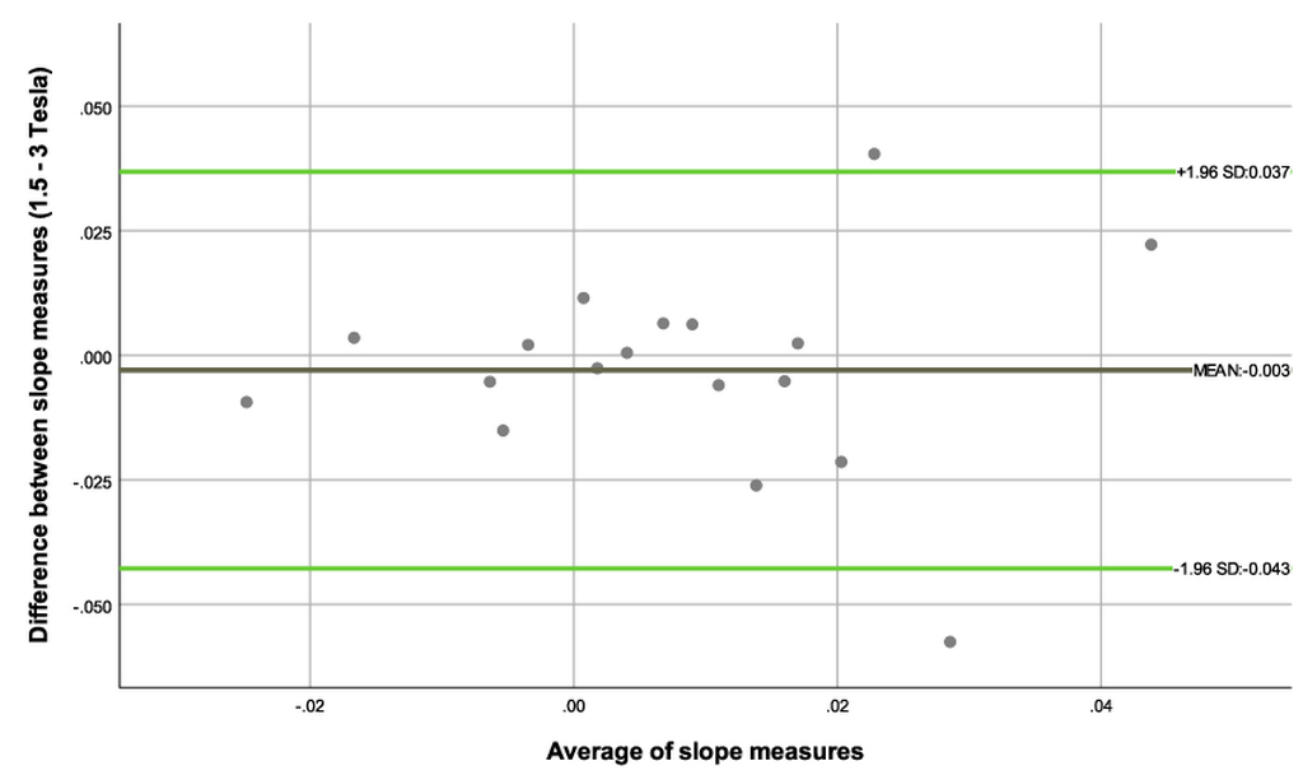

Bland-Altman plot comparing signal intensity ratio slope gradients for patients that were scanned more 3 or more times each at 1.5 and 3 Tesla using the same contrast agent. Apart from 2 outliers, all repeated measurements are within the $95 \%$ limits of agreement, and most present very small differences when measured with either a 1.5 or a 3 Tesla scanner.

$86 \times 50 \mathrm{~mm}(300 \times 300$ DPI $)$ 

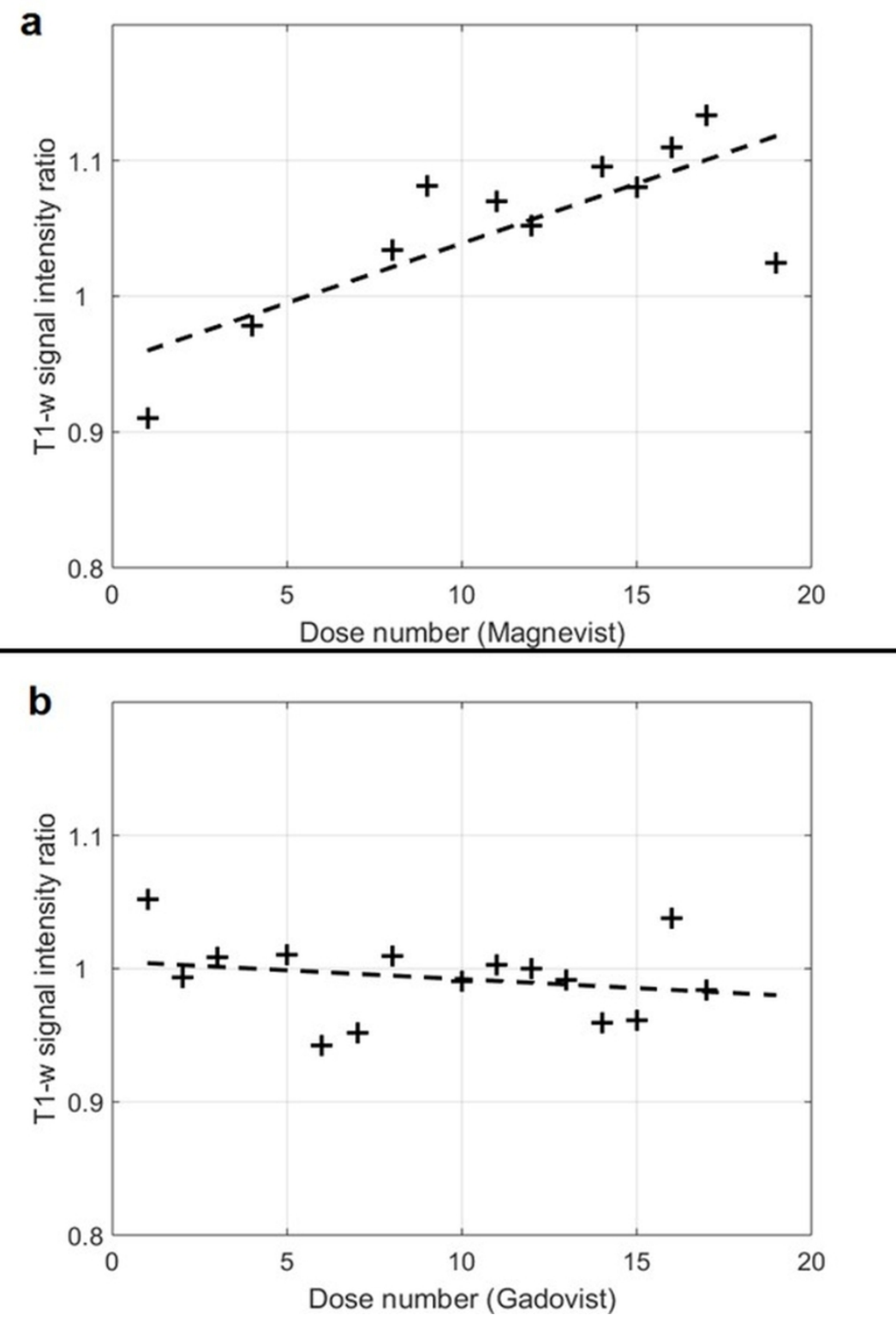

Individual subject data for relative globus pallidus (GP) / cerebral white matter (CWM) T1 signal intensity ratios for (a) a patient from the linear group and (b) a patient from the macrocyclic group. DN/MCP and GP/CWM ratios for each dose are expressed relative to the respective values derived from the unenhanced T1-weighted image prior to the first dose of gadolinium based contrast agent (GBCA).

$$
87 \times 129 \mathrm{~mm}(300 \times 300 \mathrm{DPI})
$$




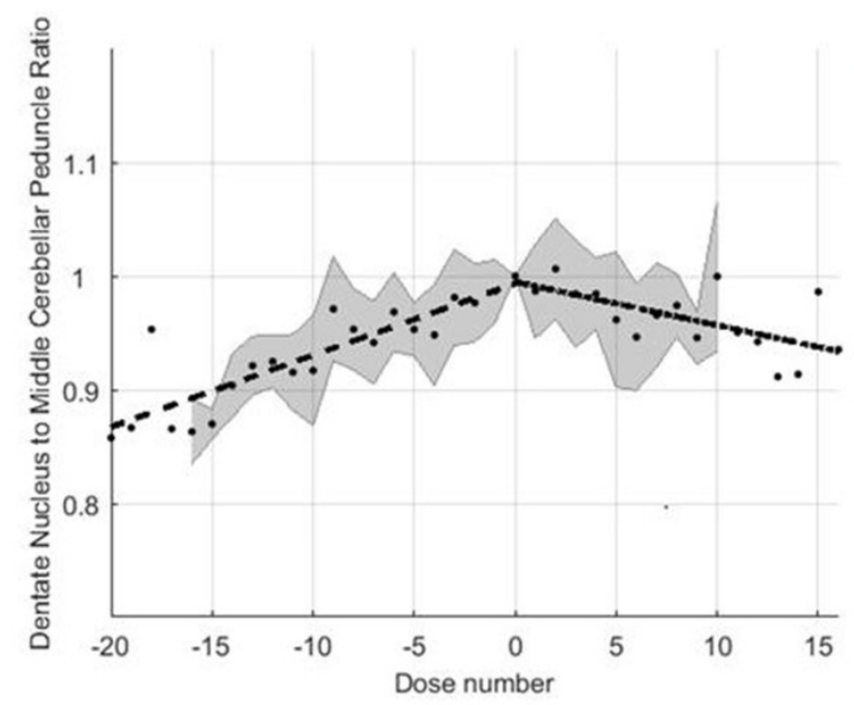

a

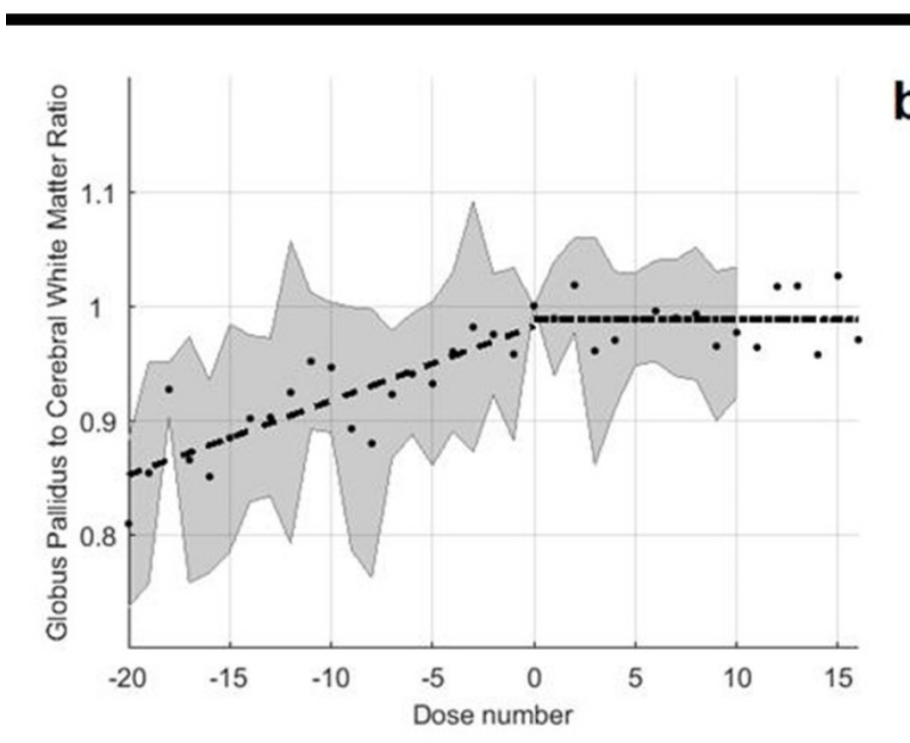

b

Group mean values $(n=18)$ for each dose and fitted slopes from the switchover group for the relative (a) dentate nucleus (DN) / middle cerebellar peduncle (MCP) and (b) globus pallidus (GP) / cerebral white matter (CWM) T1 signal intensity ratios before (dashed line) and after (dash-dot line) the switch from linear to macrocyclic. For each patient, the intensity ratios of the linear group are normalized by the ratio of the last linear contrast agent scan, and macrocyclic ratios by the ratio of their first macrocyclic scan, so that both those scans are plotted at $(0,1)$. The gray area represents the standard deviation around the mean values.

$86 \times 137 \mathrm{~mm}(300 \times 300 \mathrm{DPI})$ 
Image Quality

In a cohort of pediatric patients, it is difficult to obtain optimal image quality during MRI without using general anesthetic due to patient movement. In addition to this, flow artifact in the posterior fossa is a common feature. Many MR images were rejected for these reasons. Images were scored 0-3 where $0=$ unacceptable quality or performed elsewhere; 1 = inferior quality but acceptable in the areas of analysis; 2 $=$ acceptable quality and $3=$ optimal scan.

Supplementary Figures:

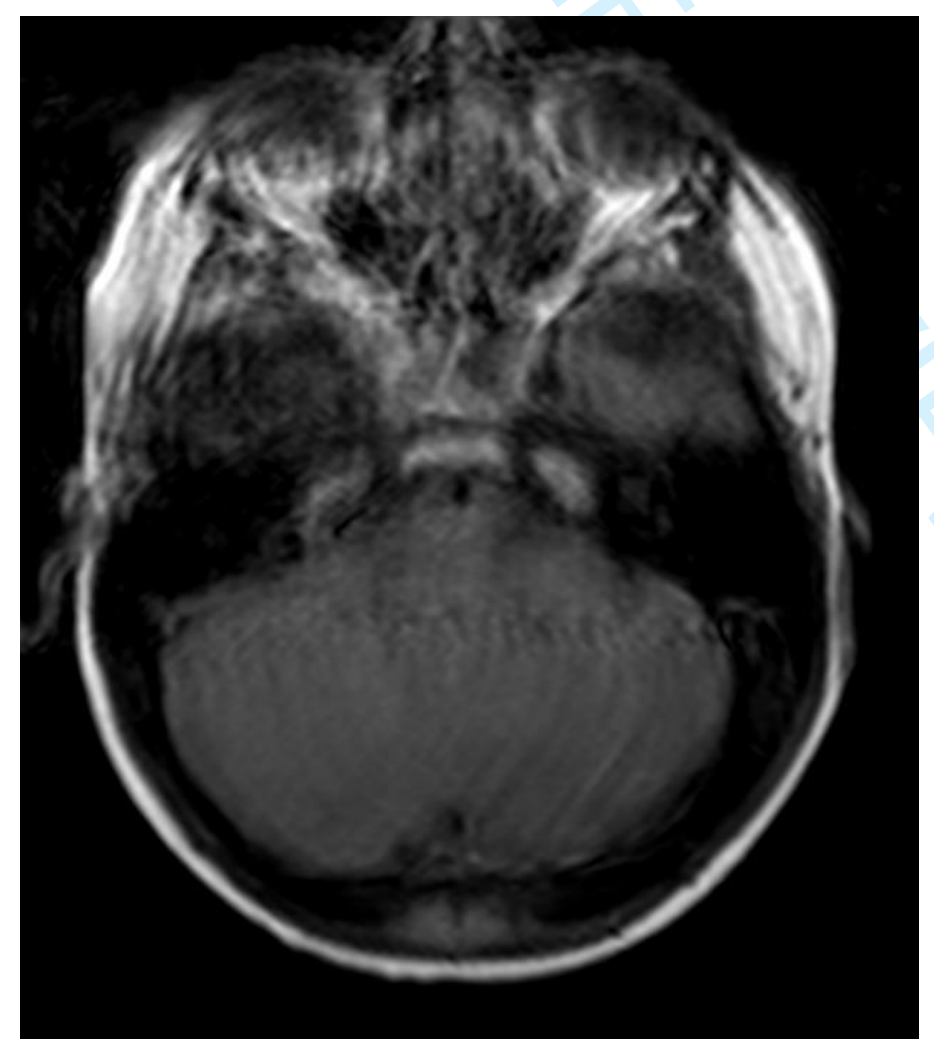

Supplementary Figure 1 -An example of category 0; pre-contrast axial T1-weighted scan with unacceptable movement artifact. 


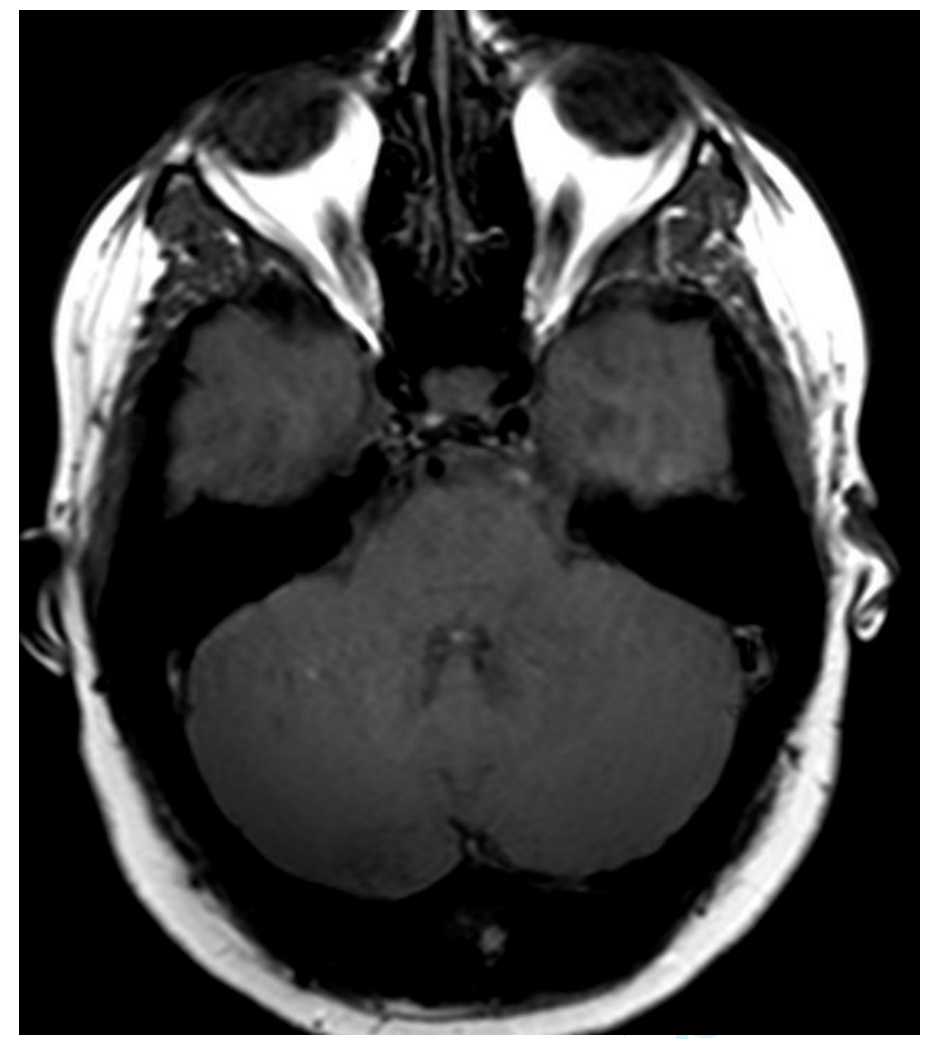

Supplementary Figure 2 - An example of category 1; pre-contrast axial T1-weighted scan with some flow artifact but it is acceptable in the lower portion of the dentate nucleus.

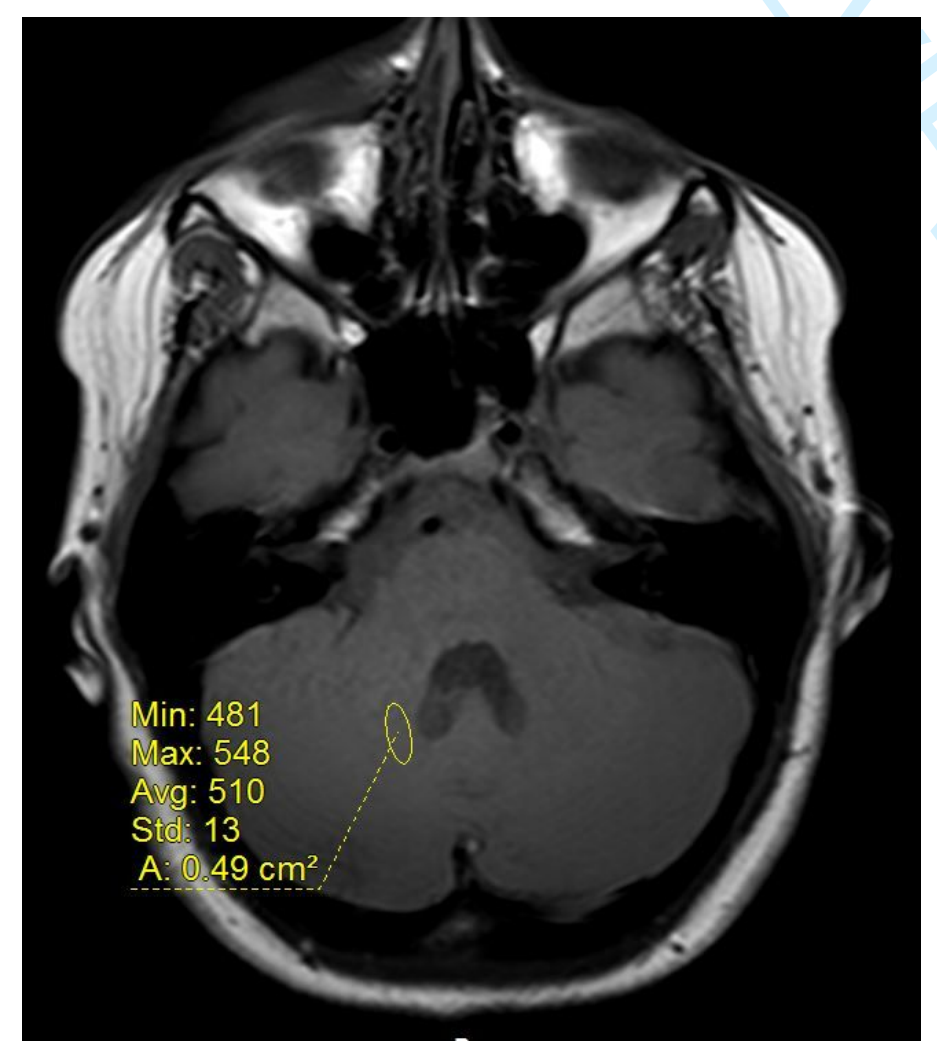

Supplementary Figure 3 - An example of category 2; axial pre-contrast T1- weighted scan of acceptable quality with minimal artifact 


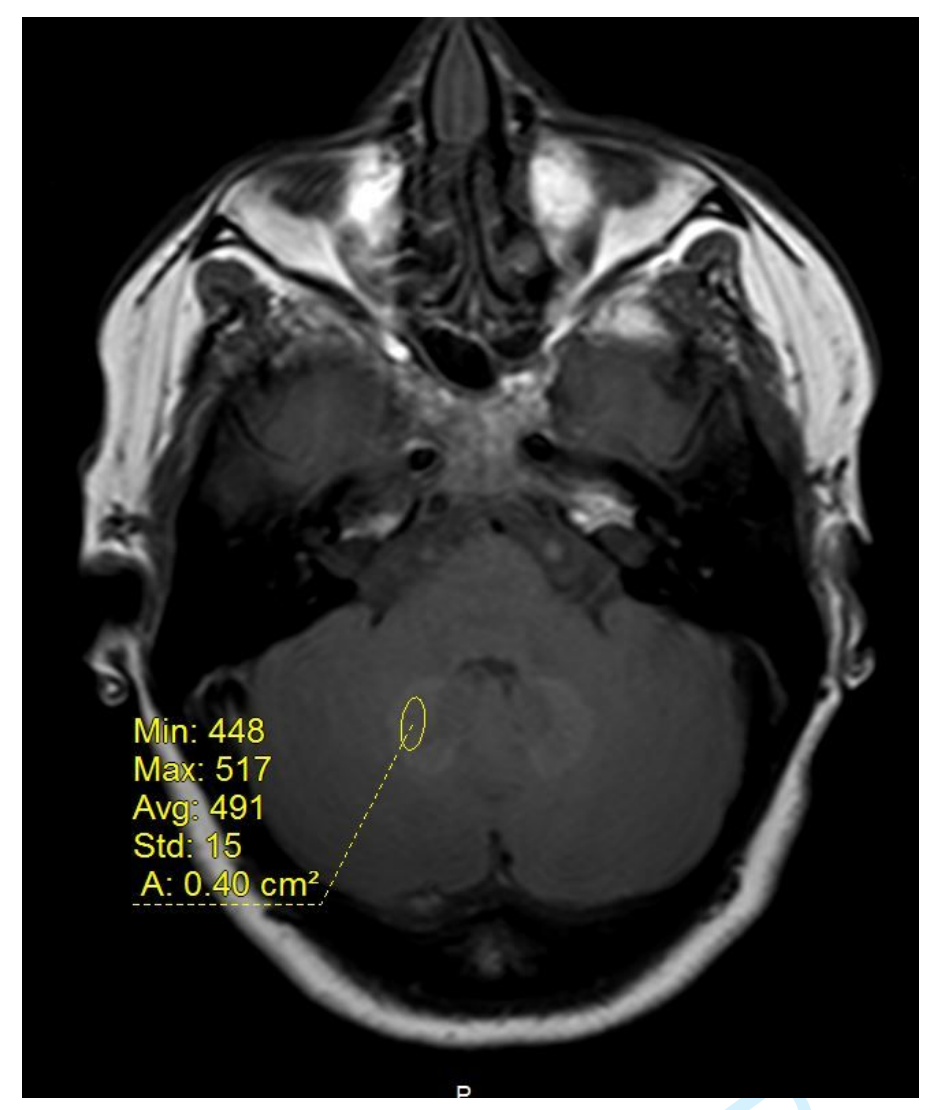

Supplementary Figure 4 - An example of category 3; axial pre-contrast T1-weighted scan of good quality 